\title{
Stability Analysis of an Assembly Process Using Simulation
}

\author{
L. Rónai ${ }^{1, *}$, T. Szabó ${ }^{1}$ \\ ${ }^{1}$ University of Miskolc, Robert Bosch Department of Mechatronics \\ Egyetemváros, 3515 Miskolc, Hungary \\ e-mail: ronai.laszlo@uni-miskolc.hu
}

Abstract: This paper deals with an assembly process of batteries with cell holder. The operation involves snap-fitting phenomenon, which is a mechanical stability problem. The structure of the cell holder is modelled with 2D flexible beam elements assuming large displacements. The stability of the equilibrium is investigated taking into consideration non-frictional and Coulomb frictional contacts. The goal of the analysis to determine the boundary point of the feed-motion from which the battery snaps-in to the final assembled position autonomously. The effect of the velocity of the battery feed-motion is also considered with energy approach.

Keywords: stability; nonlinear structure; friction; snap-fit

\section{Introduction}

Snap-fit elements [1] [2] [3] [4] are frequently applied in toy industry and also in daily used equipment. These products are assembled using human force with the help of human touch [5]. A man can sense the occurrence of the snap-fit if the process is carried out. Industrial robots are usually equipped with position control only, but it can be enhanced with machine haptic feedback [6]. Robotic assembling tasks, which involve snap-fit elements require their stability analysis in order to protect from overloading the workpiece and the robot. Another requirement is to minimize the execution time of assembling.

The stability of mechanical equilibrium containing only conservative forces can be discussed with the Lagrange-Dirichlet theorems [7] [8]. When dissipative forces are also present the Salvadori's theorem is applicable [8]. Considering friction adhesion also may take place. 
Finite Element Method (FEM) can be applied to analyse elastic structures for static and dynamic problems [9] [10] [11]. Nonlinear structures including frictions can be modelled also with FEM. The snap-fit elements are flexible structures, which can be represented with slender beam elements assuming large displacements and small deformations. A nonlinear analysis of the snap-fit can be performed by the help of Updated Lagrangian description [12] or corotational approach [13]. In ideal circumstances normal contact takes place between the contacting bodies but in real life problems the friction cannot be neglected. In this paper a quasi-static and dynamic insertion of a battery into a plastic cell holder is investigated without friction and with Coulomb friction. Snap-fit phenomenon takes place at instable equilibrium position. A numerical method is proposed in this paper to determine a so-called pre-instability position in case of dynamic assembly.

The rest of the paper is organized as follows: Section 2 describes the stability analysis of the non-friction and friction contact models. Concluding remarks are summarized in Section 3.

\section{Stability analysis of the assembling process}

Assembling operation of a battery into a cell holder by an industrial robot is shown in Fig. 1. The battery is modelled as a rigid body and the cell holder is a flexible beam structure. The diameter of the battery is $18 \mathrm{~mm}$ and its mass is $\mathrm{m}=0.0458 \mathrm{~kg}$.

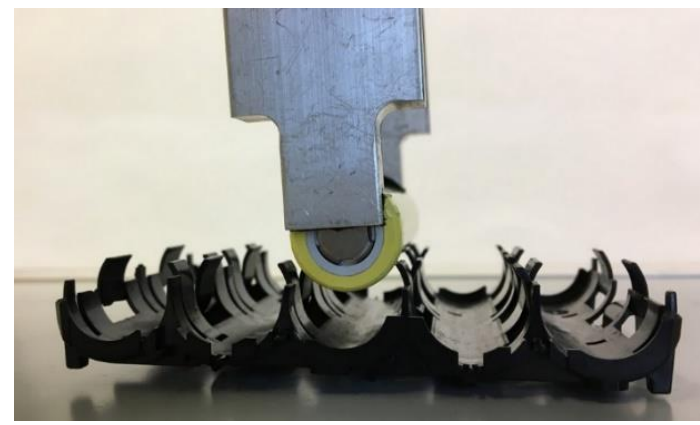

Figure 1. Assembling of a battery into a cell holder

The symmetric geometry of the curved beam structures is shown in Fig. 2. The curved beams of the cell holder consist of two arches with equal radius $\mathrm{R}=9 \mathrm{~mm}$ and the angles are $1 \mathrm{rad}$ and $0.63 \mathrm{rad}$. The cross section of the beam area is $\mathrm{A}=18.25 \mathrm{~mm}^{2}$ and the moment of inertia of cross section is $\mathrm{I}=1.52 \mathrm{~mm}^{4}$. The material of the cell holder is Acrylonitrile Butadiene Styrene (ABS) and the Young's modulus is 2.415 $\mathrm{GPa}$. One branch of the cell holder subdivided into 20 uniform beam elements. 


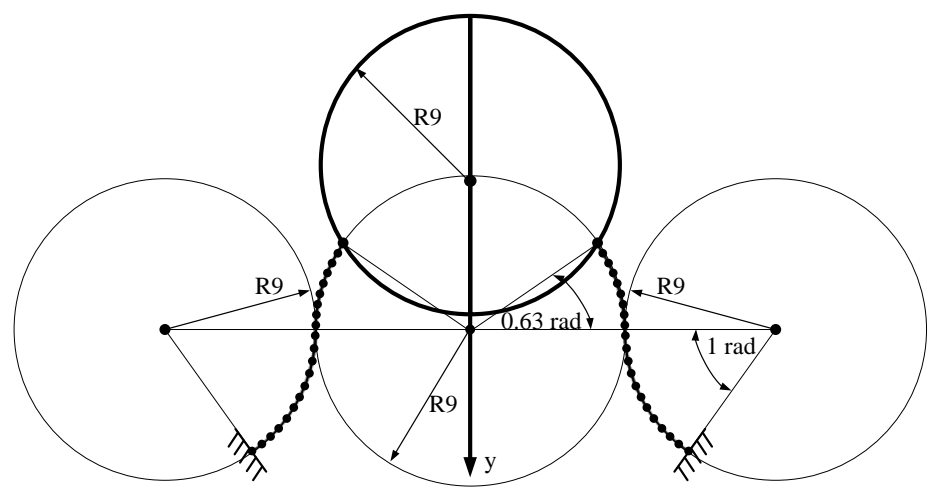

Figure 2. 2D geometry of the battery and cell holder

A special purpose nonlinear finite element program has been developed under Scilab system in order to analyse the feed-motion of the battery [6].

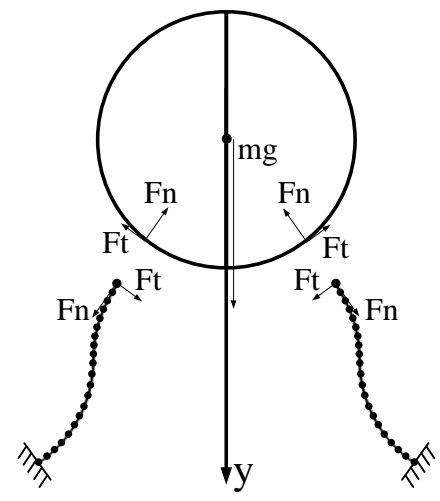

Figure 3. Free body diagram of the contact forces

The equilibrium iteration $s=1,2,3, \ldots$, is performed by Newton-Raphson method

$$
{ }^{t+\Delta t} \widehat{\mathbf{K}}_{\mathbf{t}}^{(s)} \Delta \mathbf{q}^{(s)}={ }^{t+\Delta t} \mathbf{f}_{\mathbf{u}}^{(s)}-{ }^{t+\Delta t} \mathbf{f}_{\mathbf{c}}^{(s-1)}-{ }^{t+\Delta t} \hat{\mathbf{f}}_{\mathbf{i}}^{(s-1)},
$$

where ${ }^{t+\Delta t} \widehat{\mathbf{K}}_{\mathbf{t}}^{(s)}$ is the tangential stiffness matrix, ${ }^{t+\Delta t} \mathbf{f}_{\mathbf{u}}^{(s)}$ is the kinematical load vector, ${ }^{t+\Delta t} \mathbf{f}_{\mathbf{c}}{ }^{(s-1)}$ is the force vector due to contact, ${ }^{t+\Delta t} \hat{\mathbf{f}}_{\mathbf{i}}^{(s-1)}$ is the internal load vector, and $\Delta \mathbf{q}^{(s)}$ is the vector of the displacement increments. The associated vectors and matrices of the finite elements are published in [6]. It is noted that the beam elements suffer large displacements and small deformations. 
The program determines the contact forces (see Fig. 3) in the course of feedmotion. The assembly force is the sum of the vertical projections of the normal and tangential contact forces $F_{n}, F_{t}$. It is noted that in non-friction case $F_{t}=0$.

\subsection{Modelling with non-friction contact}

The computed assembly force assuming frictionless operation is shown in Fig. 4. The feed-motion denotes the vertical displacement of the battery. The maximum displacement is $10.05 \mathrm{~mm}$ and it was performed by 30 uniform increments. The weight of the battery $0.449298 \mathrm{~N}$ is represented by horizontal thin solid line. The robotic assembly is performed very slowly, which can be regarded as a quasi-static motion. The goal is to find equilibrium position having instability, where only the gravity is exerted on the battery and for any arbitrary small disturbance it moves autonomously to the assembled position. The intersection of the curve of the load versus feed-motion and the weight of the battery provide two equilibrium points.

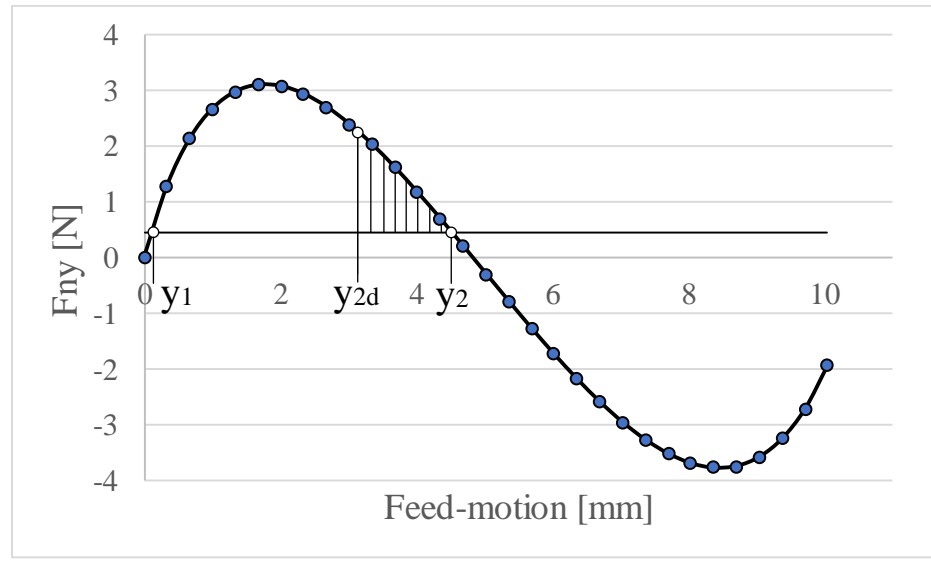

Figure 4. Insertion load versus feed-motion of the battery

The first equilibrium point is at $y_{1}=0.1178 \mathrm{~mm}$, the second one is at $y_{2}=$ $4.52552 \mathrm{~mm}$. In accordance with Lagrange-Dirichlet method the criteria of the stability is $\delta \Pi=0$ and $\delta^{2} \Pi>0$, where $\Pi$ is the potential energy of the system.

The potential energy is written as

$$
\Pi=U-m g y,
$$

where $U$ is the strain energy of the flexible cell holder.

The first variation of the potential energy as follows 


$$
\delta \Pi=0=\frac{\partial U}{\partial y} \delta y-m g \delta y=\left(\frac{\partial U}{\partial y}-m g\right) \delta y,
$$

where $\frac{\partial U}{\partial y}=F_{n y}$ represents the vertical projection of the internal force, which is equal to $m g$ at the equilibrium positions.

Taking of the second variation of the potential energy

$$
\delta^{2} \Pi=\frac{\partial^{2} U}{\partial y^{2}}(\delta y)^{2}=\frac{\partial F_{n y}}{\partial y}(\delta y)^{2},
$$

where $\frac{\partial F_{n y}}{\partial y}>0$ at $y_{1}=0.1178 \mathrm{~mm}$ and $\frac{\partial F_{n y}}{\partial y}<0$ at $y_{2}=4.52552 \mathrm{~mm}$ according to Fig. 4. Therefore, the first equilibrium point is stable and the second one is unstable. It means that if the end-effector of the robot is opened a bit over the second point, the battery would snap-in.

Usually the assembling operation is performed dynamically, which means that when the gripper is opened the battery has an initial velocity, e.g., in teach mode of the robot its magnitude $v_{0}=0.25 \mathrm{~m} / \mathrm{s}$. Therefore, the gripper can be opened earlier at $y_{2 d}$ called as pre-instable position, and its kinetic energy can cover the strain energy increment, which is equal to the area of the triangle shown in Fig. 4:

$$
\frac{1}{2} m v_{0}^{2}=\int_{y_{2 d}}^{y_{2}} F_{n y} d y-m g\left(y_{2}-y_{2 d}\right),
$$

where

$$
\int_{y_{2 d}}^{y_{2}} F_{n y} d y=\int_{0}^{y_{2}} F_{n y} d y-\int_{0}^{y_{2 d}} F_{n y} d y \cong I_{F}\left(y_{2}\right)-I_{F}\left(y_{2 d}\right),
$$

$I_{F}(y)$ is the integral of the internal force computed numerically shown in Fig. 5. 


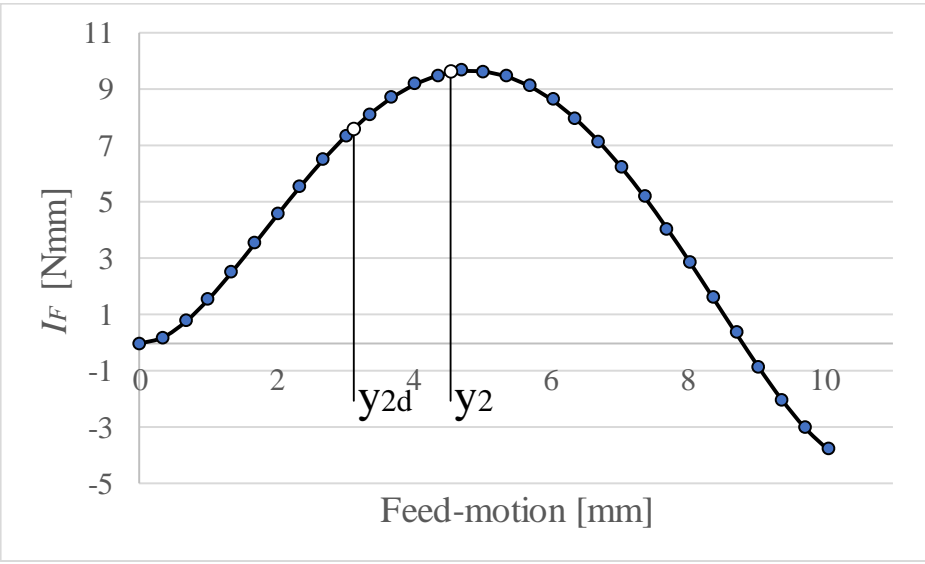

Figure 5. Numerically integrated internal force

Substituting (6) into (5) $I_{F}\left(y_{2 d}\right)$ can be expressed as:

$$
I_{F}\left(y_{2 d}\right) \cong I_{F}\left(y_{2}\right)-\frac{1}{2} m v_{0}^{2}-m g\left(y_{2}-y_{2 d}\right),
$$

the position $y_{2 d}$ can be obtained by the inverse function of $I_{F}(y)$ :

$$
y_{2 d} \cong I_{F}^{-1}\left(I_{F}\left(y_{2 d}\right)\right) \text {. }
$$

Solving numerically the implicit equation (7) $y_{2 d} \cong 3.07 \mathrm{~mm}$. The opening command of the end-effector can be initiated by the robot when the battery is arrived at the $y_{2 d}$ position then it will snap-in to its assembled position. It means that the dynamical feed-motion of the battery shorten the feed-motion and the assembling time at the same time.

\subsection{Modelling with friction}

Friction is always present in practical contact problems. Therefore, to take it into consideration in the assembling operation of the battery is obvious. Assuming Coulomb dry sliding friction the coefficient is taken to $\mu=0.15$ between the cell holder and the plastic-coated battery during feed-motion. It means that according to Fig. 3

$$
F_{t}=\mu F_{n}
$$

When the feed-motion is stopped in an equilibrium position adhesion may takes place in state of stability or sliding (9) in case of instability. 


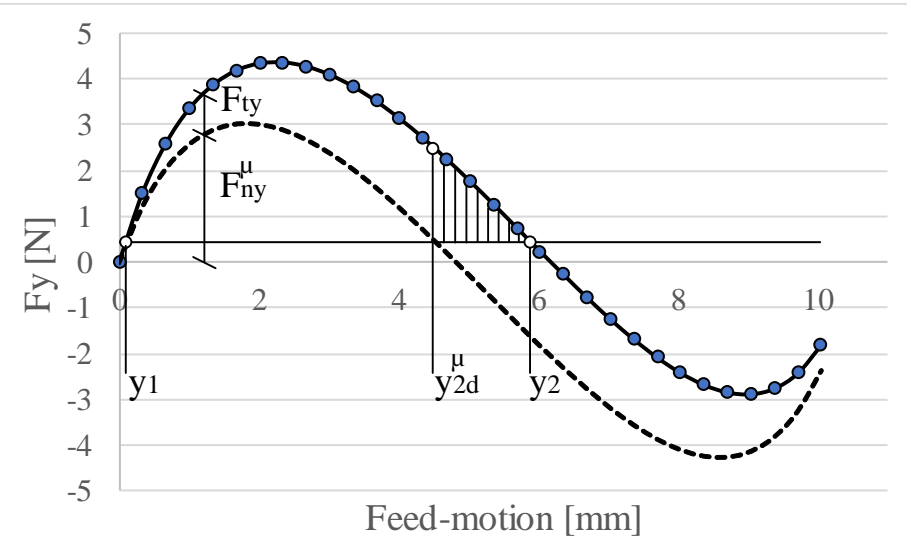

Figure 6. Load versus feed-motion curve considering friction

The vertical components of the tangential $F_{t y}$ and normal $F_{n y}^{\mu}$ contact forces have been computed by the nonlinear FEM program considering Coulomb friction (9). The results are shown in Fig. 6. It is noted that the curve of the normal contact force $F_{n y}^{\mu}$ though it is like the curve $F_{n y}$ in Fig. 4 but not equal because the principle of the superposition is not applicable for nonlinear problems. The assembly force can be obtained by the projection of the normal and tangential contact forces:

$$
F_{y}=F_{t y}+F_{n y}^{\mu} \text {. }
$$

There are also two equilibrium positions obtained by the weight of the battery. In the first equilibrium position at $y_{1}$ stick takes place and the system can be regarded to be conservative and can be treated as in Section 1.1. The second one at $y_{2}=$ $5.95 \mathrm{~mm}$ is a candidate point for the snap-fit. However, the investigated system is non-conservative due to friction. The stability of a non-conservative mechanical systems can be analysed by Salavadori's theorem.

Theorem 1 (L. Salvadori [1966] ref. [8]):

Hypothesis (i) If the potential energy $\Pi$ has a minimum at $q=0$;

Hypothesis (ii) the equilibrium at $q=0$ is isolated;

Hypothesis (iii) the dissipation is complete, i.e., for some function $a \in \mathcal{K}:(Q \mid \dot{q}) \leq-a(\|\dot{q}\|)$.

Then the equilibrium $q=\dot{q}=0$ is asymptotically stable. 
If the first Hypothesis (i) is replaced by the condition (i-a) $\Pi$ has no minimum at $q=0$ then the equilibrium $q=\dot{q}=0$ is unstable.

As it was noted previously, the equilibrium position at $y_{2}$ is a candidate for instability. Therefore, the (i-a) condition will be investigated. The notations of the theorem are given as follows $q=y-y_{2}, \dot{q}=\dot{y}$ and $Q=F_{t}$, noting that $\dot{q}$ and $F_{t}$ have opposite signs.

The potential energy of the conservative gravity and internal forces:

$$
\Pi=U-m g q \text {. }
$$

The first derivative of the potential energy is written

$$
\left.\frac{\partial \Pi}{\partial q}\right|_{q=0}=\frac{\partial U}{\partial q}-m g,
$$

where $\frac{\partial U}{\partial q}=\frac{\partial U}{\partial y}=F_{n y}^{\mu}$ denoted by dashed line in Fig. 6 and its value is equal to $1.625 \mathrm{~N}$, i.e.,

$$
\left.\frac{\partial \Pi}{\partial y}\right|_{y=y_{2}}=F_{n y}^{\mu}-m g=-1.625-0.4493 \neq 0 .
$$

The instability of the candidate position $y_{2}$ is proved mathematically according to Hypothesis (i-a).

Due to dynamic feed-motion $v_{0}=0.25 \mathrm{~m} / \mathrm{s}$ the gripper can be opened before the battery arrives at position $y_{2}$ also in case of friction. The determination of the preinstable position $y_{2 d}^{\mu}$ is performed by a similar method, which was detailed in nonfriction contact model.

$$
\frac{1}{2} m v_{0}^{2}=\int_{y_{2 d}^{\mu}}^{y_{2}} F_{y} d y-m g\left(y_{2}-y_{2 d}^{\mu}\right),
$$

where

$$
\int_{y_{2 d}^{\mu}}^{y_{2}} F_{y} d y=\int_{0}^{y_{2}} F_{y} d y-\int_{0}^{y_{2 d}^{\mu}} F_{y} d y \cong I_{F}^{\mu}\left(y_{2}\right)-I_{F}^{\mu}\left(y_{2 d}^{\mu}\right),
$$

$I_{F}^{\mu}(y)$ is the numerically computed integral of the internal force shown in Fig. 7. 


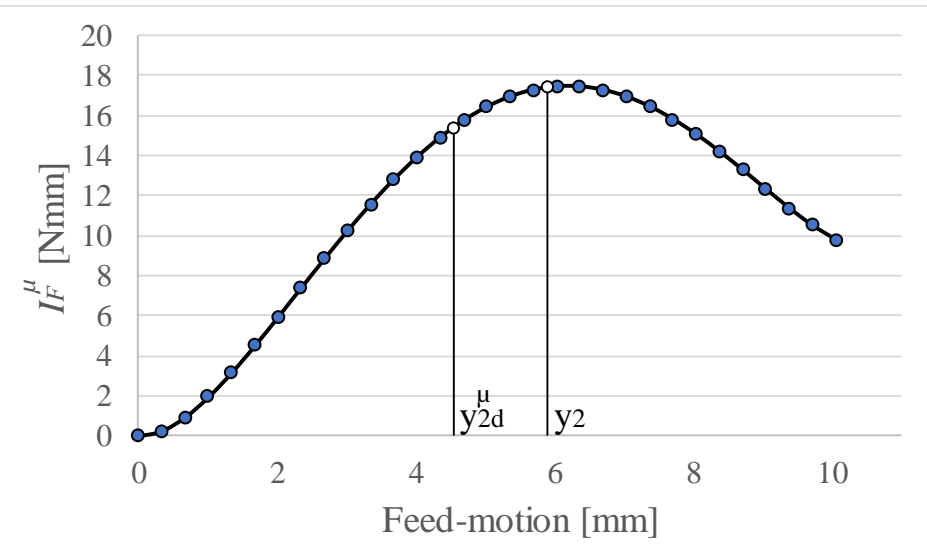

Figure 7. Numerically integrated internal force considering friction

Substituting (15) into (14) the integral of the internal force at position $y_{2 d}^{\mu}$ is given as

$$
I_{F}^{\mu}\left(y_{2 d}^{\mu}\right) \cong I_{F}^{\mu}\left(y_{2}\right)-\frac{1}{2} m v_{0}^{2}-m g\left(y_{2}-y_{2 d}^{\mu}\right),
$$

$y_{2 d}^{\mu}$ can be determined from (8) by iteration. The gripper can be opened at position $y_{2 d}^{\mu}=4.522 \mathrm{~mm}$ earlier than $y_{2}$.

\section{Conclusions}

Stability analysis of a nonlinear system containing battery and cell holder has been described in this paper. Two equilibrium positions were investigated with nonfriction and friction contact models. The flexible elastic nonlinear structure was modelled with slender beam FEM elements.

Stable and unstable equilibrium positions have been determined assuming quasi static feed-motion. The latter one produces snap-fit phenomenon, where a robot gripper can be opened, then the assembly is performed autonomously.

An approximate method is proposed in this paper to determine a pre-instable position before the equilibrium is reached. It is shown that the distance of the feedmotion can be decreased when the kinetic energy of the battery is not neglected, i.e., dynamical assembly is performed with a given velocity. 


\section{Acknowledgement}

The described article was carried out as part of the EFOP-3.6.1-16-2016-00011 "Younger and Renewing University - Innovative Knowledge City - institutional development of the University of Miskolc aiming at intelligent specialisation" project implemented in the framework of the Széchenyi 2020 program. The realization of this project is supported by the European Union, co-financed by the European Social Fund.

\section{References}

[1] BASF Corporation, Snap-Fit Design Manual (2007) [cited 2020-01-04] URL www.plasticsportal.com/usa

[2] BAYER Materialscience LLC, Snap-Fit Joints for Plastics, A design Guide, Bayer Polycarbonates Business Unit, Pittsburg, Pennsylvania, 1998.

[3] C. Klahn, D. Singer, M. Meboldt, Design Guidelines for Additive Manufactured Snap-Fit Joints, Procedia CIRP 50 (2016) pp. 264-269. doi: https://doi.org/10.1016/j.procir.2016.04.130

[4] R. M. Kshirsagarl, D. B. Pawar, Design and analysis of snap fit joint in plastic part, International Journal of Innovative and Emerging Research in Engineering 2 (1) (2015) pp. 83-87.

[5] M. Radi, G. Reinhart: Industrial Haptic Robot Guidance System for Assembly Processes, IEEE Conference, Lecco, Italy, 2009, pp.1-6. doi: https://doi.org/10.1109/HAVE.2009.5356135

[6] L. Rónai, T. Szabó, Snap-fit Assembly Process with Industrial Robot Including Force Feedback, Robotica, Cambridge University Press 38 (2) (2020) pp.317-336. doi: https://doi.org/10.1017/S0263574719000614

[7] Gy. Béda, I. Kozák, Mechanics of elastic bodies, Müszaki könyvkiadó, Budapest, 1987, in Hungarian.

[8] N. Rouche, P. Habets, M. Laloy, Stability Theory by Liapunovs Direct method, Springer-Verlag, New York, 1977. 
[9] L. Fehér L, J. Égert, FEM Modeling and Weight Reduction of a Solar Energy Driven Racing Car Chassis, Acta Technica Jaurinensis 8 (4) (2015) pp. 296311.

doi: https://doi.org/10.14513/actatechjaur.v8.n4.385

[10] J. Égert, Finite Element Mechanical Modeling Opportunities in Machine Design, Acta Technica Jaurinensis 1 (1) (2008) pp. 47-59.

[11] P. Horváth, J. Égert, Dynamic Analysis of a One-cylinder Engine Crankshaft, Acta Technica Jaurinensis 8 (4) (2015) pp. 280-295.

doi: https://doi.org/10.14513/actatechjaur.v8.n4.379

[12] K. J. Bathe: Finite Element Procedures, Prentice Hall, Upper Saddle River, New Jersey, 1996.

[13] M. A. Crisfield: Non-linear Finite Element Analysis of Solids and Structures, John Wiley and Sons, New York City, 1991. 